OPEN

SUBJECT AREAS:

ATHEROSCLEROSIS

MONOCYTES AND

MACROPHAGES

MECHANISMS OF DISEASE

CHRONIC INFLAMMATION

Received

18 October 2012

Accepted

29 November 2012

Published

16 January 2013

Correspondence and requests for materials should be addressed to

J.L.M. (MehtaJL@

UAMS.edu)

* These authors

contributed equally to

this paper.

\section{Oxidant stress in mitochondrial DNA damage, autophagy and inflammation in atherosclerosis}

\author{
Zufeng Ding*, Shijie Liu*, Xianwei Wang, Magomed Khaidakov, Yao Dai \& Jawahar L. Mehta
}

Central Arkansas Veterans Healthcare System and the Department of Medicine, University of Arkansas for Medical Sciences, Little Rock, Arkansas.

Our studies in HUVECs show that ox-LDL induced autophagy and damaged mtDNA leading to TLR9 expression. LOX-1 antibody or the ROS inhibitor apocynin attenuated ox-LDL-mediated autophagy, mtDNA damage and TLR9 expression, suggesting that these events are LOX-1 and ROS-dependent phenomena. Experiments using siRNA to DNase II indicated that DNase II digests mtDNA to protect the tissue from inflammation. Next, we studied and found intense autophagy, TLR9 expression and inflammatory signals (CD45 and CD68) in the aortas of LDLR knockout mice fed high cholesterol diet. Deletion of LOX-1 (LDLR/LOX-1 double knockout mice) attenuated autophagy, TLR9 expression as well as CD45 and CD68. Damaged mtDNA signal was also very high in LDLR knockout mice aortas, and this signal was attenuated by LOX-1 deletion. Thus, it appears that oxidative stress-mediated damaged mtDNA that escapes autophagy induces a potent inflammatory response in atherosclerosis.

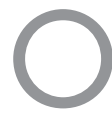

xidized-low density lipoproteins (ox-LDL) accumulate in the arterial wall and activate signaling pathways that lead to inflammation and oxidative stress response ${ }^{1}$. Lectin-like oxidized low-density lipoprotein scavenger receptor-1 (LOX-1) is one of the major receptors responsible for binding, internalizing and degrading ox-LDL ${ }^{2}$. Activation of LOX-1 has been known to be related to many pathophysiological events, including endothelial cell and vascular smooth muscle cell proliferation, alteration in cell cycle signals, apoptosis and autophagy ${ }^{1-4}$.

As energy-producing organelles, mitochondria can suffer damage under oxidative stress that induces endothelial dysfunction and promotes leukocyte adhesion, inflammation, thrombosis and smooth muscle cell proliferation $^{5}$. Damaged mitochondria are often degraded by autophagy, which is an evolutionarily conserved process for lysosomal recycling of cytoplasmic material ${ }^{6}$. Similar to bacterial DNA, mitochondrial DNA (mtDNA) contains inflammatogenic unmethylated $\mathrm{CpG}$ motifs, whereas nuclear DNA is modified by the addition of methyl groups on certain sequences known as CpG motifs ${ }^{6,7}$. This differential feature allows immune cells to recognize DNA of invading bacteria. mtDNA activates toll-like receptor 9 (TLR-9) that senses unmethylated CpG motifs and induces the synthesis of pro-inflammatory cytokines ${ }^{8}$.

Although mtDNA released from dying cells induces a TLR9-dependent inflammatory response ${ }^{6-8}$, there is no evidence to support that TLR9 pathway is relevant in atherosclerotic plaques that exhibit extensive inflammation in the absence of infection.

We hypothesized that DNA released during autophagy in endothelial cells (ECs) could trigger an inflammatory response similar to that generated by bacterial DNA during an infection. The present study was designed to test this hypothesis. We show for the first time autophagy, mtDNA release and TLR-9 expression in cultured human ECs exposed to ox-LDL. We also show that autophagy, mtDNA release, TLR9 expression and inflammatory response in atherosclerotic regions of LDLR-null mice aortas and the attenuation of these phenomena by LOX-1 abrogation.

\section{Results}

Ox-LDL induces autophagy and ROS generation. As observed previously ${ }^{9}$, LOX-1 expression in HUVECs increased in response to increasing ox-LDL concentration $(0-60 \mu \mathrm{g} / \mathrm{ml}$, incubation time $24 \mathrm{~h}$ ) (Figure 1A top panel). We assessed autophagy in response to ox-LDL by measuring beclin-1 and LC3-I/LC3-II, both are recognized autophagosome markers $^{10}$. As shown in Figure 1A, ox-LDL (20 to $40 \mu \mathrm{g} / \mathrm{ml}$ ) treatment 
A.
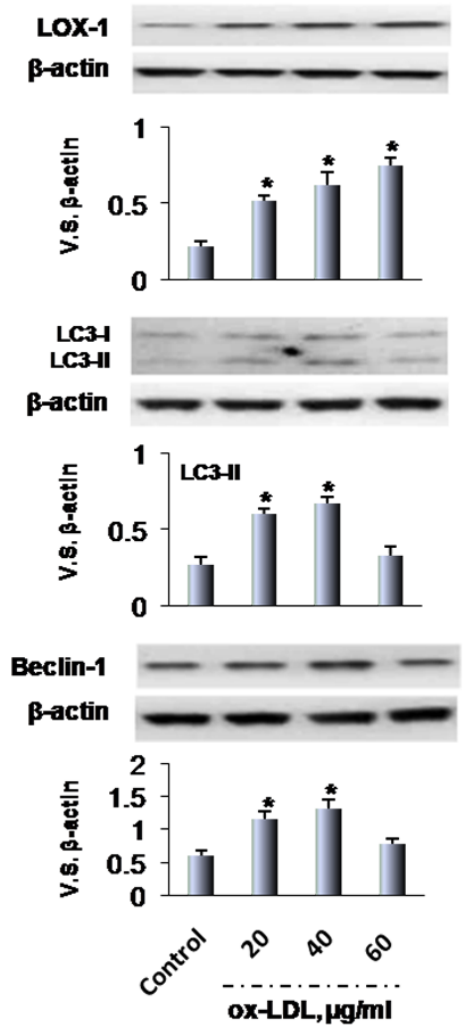

B.
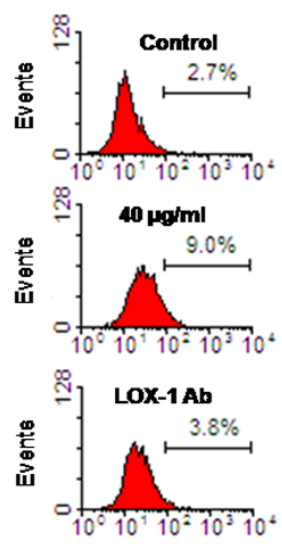

C. FL2-H

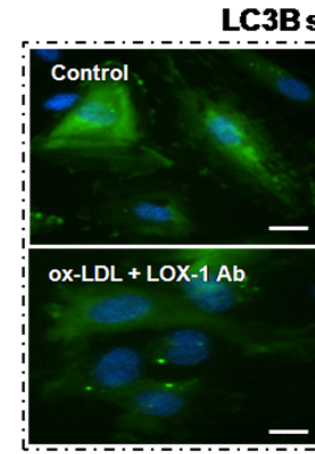

D.
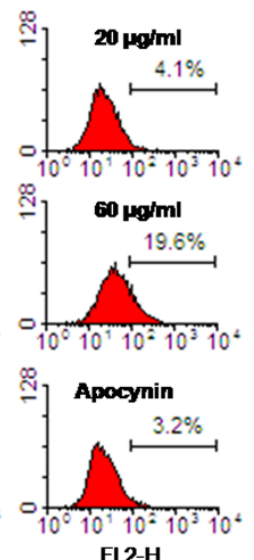

FL2-H

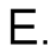

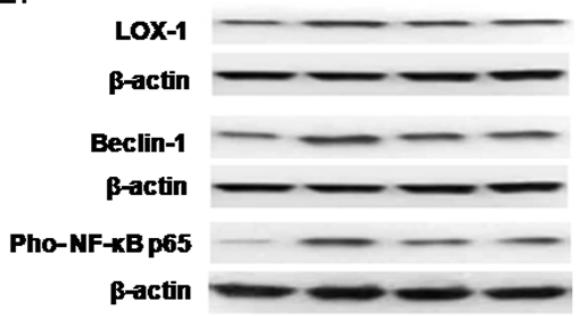

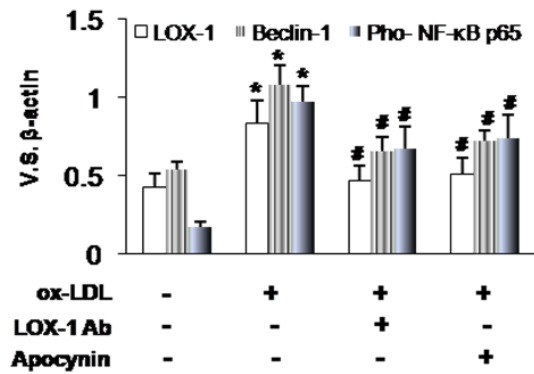

Figure $1 \mid$ Ox-LDL induces LOX-1, autophagy and ROS generation. (A) Ox-LDL induces LOX-1 expression in a dose-dependent manner. Ox-LDL (20 to $40 \mu \mathrm{g} / \mathrm{ml}$ concentrations) induces expression of LC3 and beclin- 1 ; however, $60 \mu \mathrm{g} / \mathrm{ml}$ ox-LDL decreases LC3 and beclin-1. (B) Ox-LDL in a dosedependent manner induces ROS generation which is blocked by LOX-1 Ab and apocynin. (C) Immunofluorescence shows that ox-LDL enhances LC3B (fluorescent staining) which is blocked by LOX-1 Ab and apocynin. Scale bars: $20 \mu \mathrm{m}$. (D) Ox-LDL enhances LC3B expression (flow cytometry).

(E) Ox-LDL increases the expression of LOX-1, beclin-1 and pho-NF-kB, and this effect can be blocked by LOX-1 Ab as well as apocynin. Bar graphs represent data in mean $\pm \mathrm{SD}$ based on 3-5 experiments, ${ }^{*} \mathrm{P}<0.05$ vs. Control, ${ }^{*} \mathrm{P}<0.05$ vs. ox-LDL.

significantly increased beclin-1 and LC3-II. Higher concentration of ox-LDL $(60 \mu \mathrm{g} / \mathrm{ml})$, however, decreased both autophagy markers (Figure 1A middle and lower panels).

Ox-LDL is a potent inducer of ROS, and this was confirmed in the present study (Figure 1B). Further, pretreatment of HUVECs with LOX-1 Ab or the NADPH oxidase inhibitor apocynin significantly inhibited ROS generation in response to ox-LDL (Figure 1B).

As noted previously, some of the LC3 dissociates from the membrane after fusion with the lysosome during development of autophagy, and the LC3-GFP can serve as autolysosome and as a specific marker for autophagy ${ }^{9,10}$. We examined LC3B-GFP changes in HUVECs by fluorescence microscopy (Figure 1C). In normal cells, LC3B-GFP protein was distributed diffusely throughout the entire cell with almost no LC3B accumulation in the lysosomes. After incubating HUVECs with ox-LDL for $24 \mathrm{~h}$, a large number of cells showed LC3B-GFP accumulation, suggesting activation of autophagic response. Pretreatment of cells with LOX-1 Ab or apocynin decreased LC3B accumulation induced by ox-LDL. The data from flow cytometry (Figure 1D) confirmed the results of fluorescence microscopy. Similarly to LC3B data, expression of LOX-1, beclin-1 and Pho-NF-kB p65 in cells treated with ox-LDL was inhibited by pretreatment with LOX-1 Ab or apocynin (Figure 1E).

Ox-LDL dependent mtDNA damage and regulation of TLR9. Recent clinical studies show accumulation of mtDNA in atherosclerotic lesions ${ }^{5}$. There is also evidence for accumulation of ox-LDL in atherosclerotic tissues ${ }^{1-4}$. However, there is no direct evidence of correlation of ox-LDL and mtDNA that escapes autophagy. We looked for and found that ox-LDL in a dosedependent manner induced mtDNA leakage in HUVECs (Figure 2). Further, pretreatment of cells with LOX-1 Ab or apocynin reduced mtDNA leakage (Figure 2A). PicoGreen staining based flow cytometry also confirmed the presence of mtDNA leakage (Figure 2B) and the data were similar to the immunocytochemistry observations.

Localized to intracellular compartments, TLR9 recognizes viral and bacterial DNA that contains unmethylated $\mathrm{CpG}$ dinucleotides, which induces the production of pro-inflammatory cytokines ${ }^{8}$. We examined the relationship between ox-LDL and TLR9. As shown in Figure 2C, ox-LDL (20 to $40 \mu \mathrm{g} / \mathrm{ml}$ ) increased TLR9 expression. Higher concentration of ox-LDL $(60 \mu \mathrm{g} / \mathrm{ml})$, however, reduced TLR9 expression. As with mtDNA leakage data, pretreatment with LOX-1 Ab or apocynin blocked TLR9 expression in response to oxLDL $(40 \mu \mathrm{g} / \mathrm{ml})$.

As further evidence for the link between mtDNA leakage and TLR9 expression, we treated HUVECs with 3-methyladenine (an autophagy inhibitor) or rapamycin (an autophagy inducer). We observed that 3-methyladenine enhanced and rapamycin inhibited TLR9 expression, indicating that mtDNA that escapes autophagy cell-autonomously leads to TLR9 expression.

Deoxyribonuclease II (DNase II) inhibition induces autophagy and TLR9 expression. DNases are found in the lysosome $e^{7,8}$. DNase II in particular has an essential role in the degradation of DNA of apoptotic cells after macrophages engulf them ${ }^{11}$. We hypothesized that DNase II in HUVECs would digest mtDNA and protect the 
A.

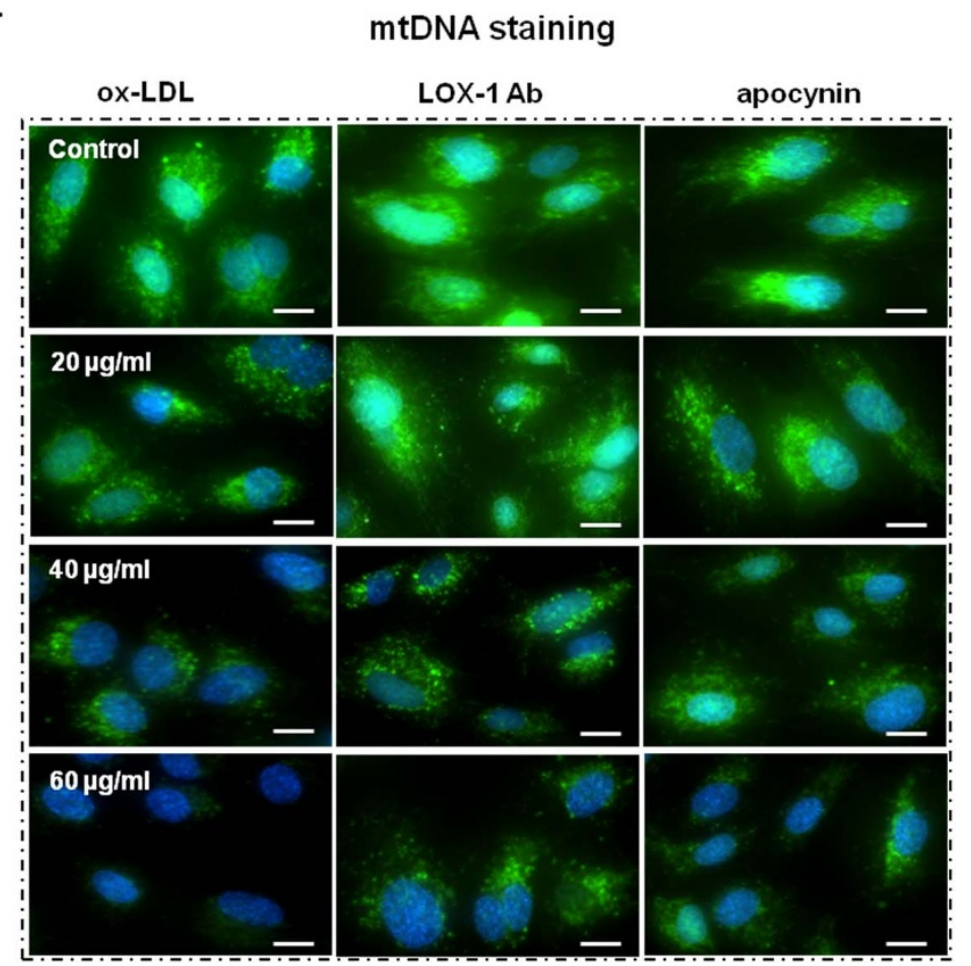

B.

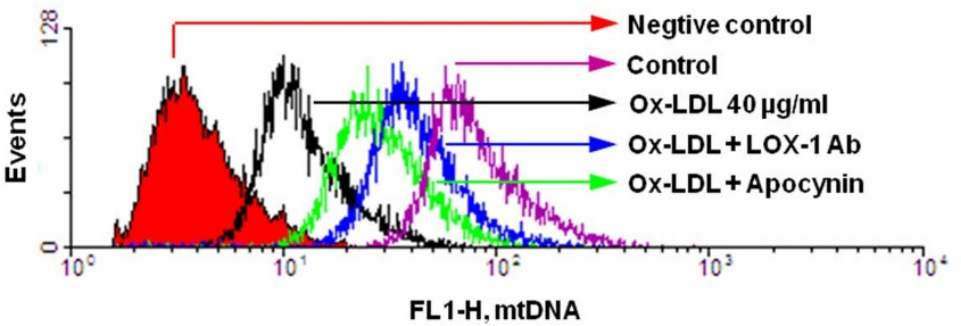

C.

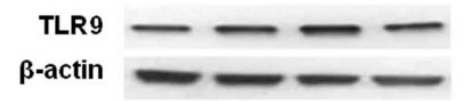

D.
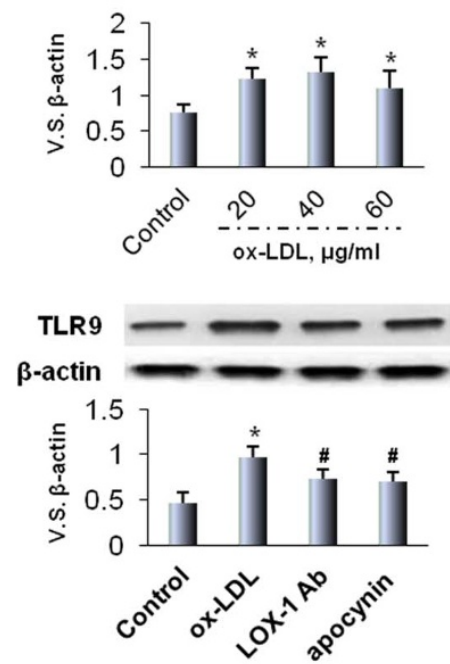

E.
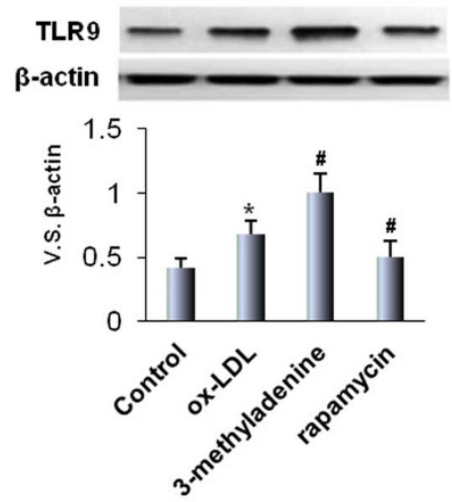

Figure 2 Ox-LDL damages mtDNA and induces TLR9 expression. (A) Ox-LDL damages mtDNA in a dose-dependent manner, and LOX-1 Ab as well as apocynin block the effect of ox-LDL. Scale bars: $20 \mu \mathrm{m}$. (B) The results of mtDNA staining are confirmed by flow cytometry. In addition, LOX-1 Ab as well as apocynin block the formation of mtDNA. (C) Ox-LDL (20 to $40 \mu \mathrm{g} / \mathrm{ml})$ induces TLR9 expression. (D) The effects of ox-LDL on the expression of TLR9 are blocked by LOX-1 Ab as well as apocynin. (E) 3-methyladenine (an autophagy inhibitor) enhances and rapamycin (an autophagy inducer) inhibits TLR9 expression. Bar graphs represent data in mean \pm SD based on 3-5 experiments, * $\mathrm{P}<0.05$ vs. Control, ${ }^{*} \mathrm{P}<0.05$ vs. ox-LDL.

cultured cells from inflammatory response (TLR9 expression) to oxLDL. To confirm this hypothesis, HUVECs were transfected with DNase II siRNA and the cells were then exposed to ox-LDL. As shown in Figure 3A and 3B, inhibition of DNase II induced LC3 and beclin-1 expression in response to ox-LDL. Consistent with our hypothesis, inhibition of DNase II also induced TLR9 expression (Figure 3C). These observations support previous data on ox-LDL induced autophagy and TLR9 expression ( Figure 1 and 2).

LOX-1 deletion reduces autophagy and TLR9 mediated inflammation response. Previous studies from our laboratory ${ }^{3}$ showed that both LDL-cholesterol and ox-LDL were higher in the LDLR KO and double KO mice than corresponding controls (WT and LOX-1 KO mice, respectively). The extent of atherosclerosis was also reduced by LOX-1 abrogation in the LDLR KO mice.

To further study the relationship between autophagy, mtDNA leakage and TLR9 in atherosclerosis, we checked atherosclerotic aorta sections from different groups of mice (Figure 4). Immunohistochemistry showed a very high expression of LC3 and beclin-1 in LDLR KO mice compared with WT mice. Concomitant with increased autophagy, mtDNA leakage and TLR9 were highly expressed in the LDLR KO mice aortas. The abrogation of LOX-1 in
LDLR KO mice (double KO mice) reduced autophagy, mtDNA leakage and TLR9 expression in the LDLR KO mice aortas. Of note, autophagy markers (LC3 and beclin-1), mtDNA leakge and TLR9 expression all were lower in LOX-1 KO mice than in the WT mice aortas.

Next, we evaluated inflammatory cell infiltration using antibodies against CD45 and CD68 (common leukocyte antigens) as markers for macrophages and leukocytes, respectively ${ }^{7}$. Immunohistochemistry showed that CD45 and CD68 were highly expressed in the LDLR KO mice aortas and their expression was reduced by LOX-1 abrogation (double KO mice). Consistent with TLR9 data, CD45 and CD68 expression was much less in the LOX-1 KO mice compared with WT mice aorta.

\section{Discussion}

Ox-LDL transport into the subendothelial space at the sites of endothelial damage is considered a seminal initiating event for atherogenesis $^{12}$. LOX-1 is present in ECs in much greater amount than any other scavenger receptor and serves to activate these cells and internalize ox-LDL. The role of LOX-1 as a key scavenger receptor became evident is studies showing significant abrogation of atherosclerosis in the LDLR KO mice ${ }^{3}$. 
A.
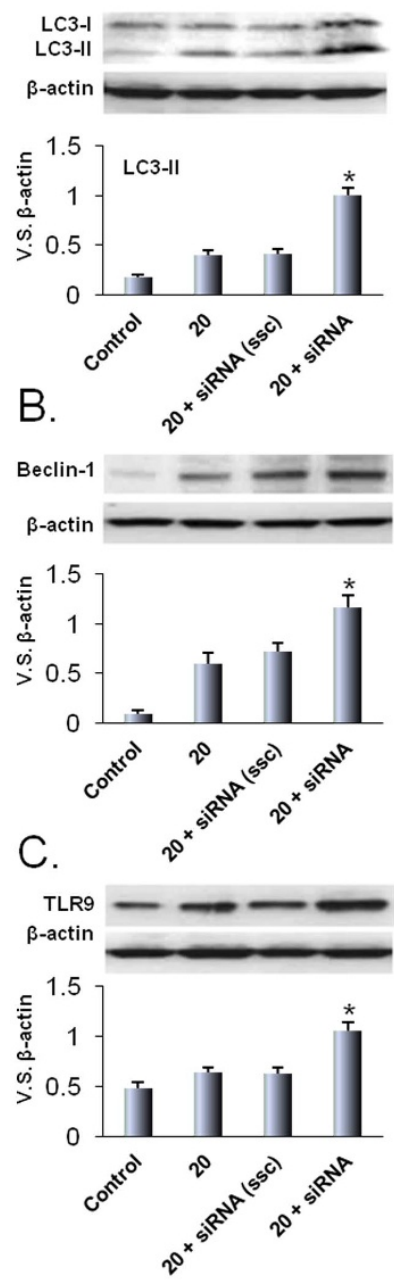

LC3 expression

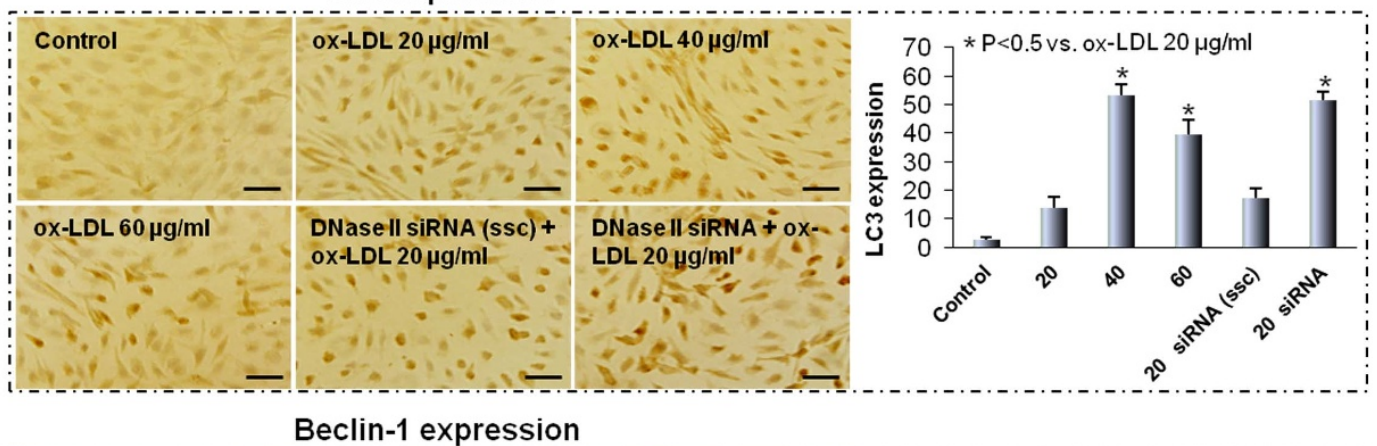

Figure 3 DNase II inhibition induces autophagy and TLR9 expression. Western blot and immunohistochemical analyses indicate that ox-LDL

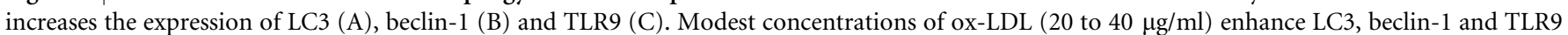

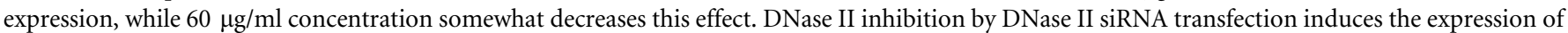

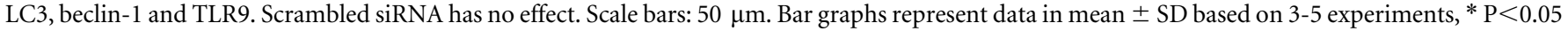
vs. ox-LDL $20 \mu \mathrm{g} / \mathrm{ml}$.

Autophagy is a potent adaptive mechanism to protect cells from stress-induced injury, which removes superfluous and damaged mitochondrial organelles ${ }^{13}$. Studies have shown that mtDNA released from dying cells into the extracellular space induces a TLR9-dependent inflammatory response in some cells of the immune system ${ }^{14,15}$. However, the mechanism of TLR9 pathway involved in atherosclerotic areas that exhibit extensive inflammation in the absence of infection has not been well studied. In the present study, we show that mtDNA leakage that escapes from autophagy leads to TLR-9 mediated inflammatory responses in cultured HUVECs. We also show that mtDNA leakage and subsequent TLR9 expression are LOX-1 mediated phenomena.

Autophagy is an evolutionary conserved process involved in the degradation of long-lived proteins, which becomes manifest during state of increased stress, and may be considered a cell survival mechanism $^{16,17}$. We now show that HUVECs when exposed to $20 \mu \mathrm{g} / \mathrm{ml}$ of ox-LDL show autophagy (measured as expression of LC3 and beclin-1), suggesting that autophagy in HUVECs in response to circulating concentrations of ox-LDL reflects a stress response that allows HUVECs to survive during harsh conditions. Importantly, in the present study the ox-LDL induced autophagy was blocked by apocynin as well as LOX-1 antibody suggesting that the autophagic response in HUVECs is mediated by ROS-LOX-1 pathway. Atherosclerosis involves oxidative stress and inflammation ${ }^{3,4}$. Keeping with this concept we found that the expression of NF- $\mathrm{KB}$ was reduced by pretreatment with LOX-1 Ab and apocynin, indicating that ROS-LOX-1 pathway is important in the expression of this transcription factor.

TLR9, localized in the endolysosome, senses DNA with unmethylated CpG motifs derived from bacteria and viruses, which is the major contributor to acute inflammation induced by microbial infection ${ }^{18,19}$. To investigate the role of ox-LDL in mtDNA mediated TLR9 expression, HUVECs were colabeled with green-fluorescing PicoGreen and Hoechst 33342. PicoGreen is a DNA-intercalating fluorophore that penetrates mitochondrial membranes and labels mtDNA, while Hoechst 33342 stains the nuclei ${ }^{20,21}$. Our data show that ox-LDL in a dose-dependent manner damaged mtDNA expression; importantly, pretreatment of cells with LOX-1 Ab or apocynin blocked this ox-LDL effect. These effects of ox-LDL on mtDNA damage were assessed by two different methods, immunocytochemistry and flow cytometery, and both methods gave similar results.

Ox-LDL induced TLR9 expression at modest concentrations (20 nd $40 \mu \mathrm{g} / \mathrm{ml}$ ) but inhibited it at $60 \mu \mathrm{g} / \mathrm{ml}$ concentration. It is likely that high concentartions of ox-LDL resulted in death of some cells resulting in lower TLR9 expression; a similar phenomenon was reported initially by Mehta et al while studying ox-LDLmediated LOX-1 expression ${ }^{2-4}$. Importantly, treatment of cells with 


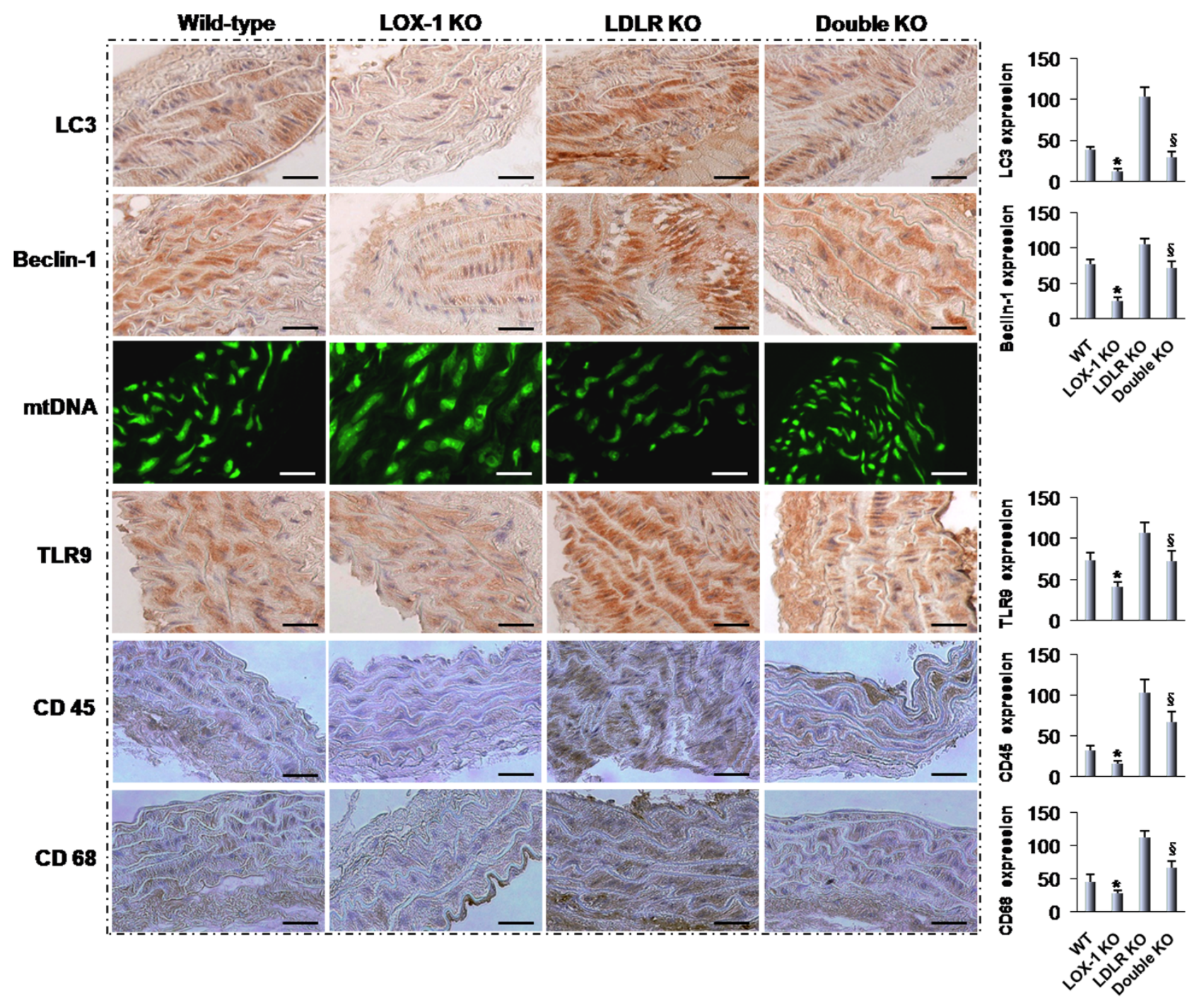

Figure $4 \mid$ Immunohistochemistry markers of autophagy, mtDNA damage, and inflammation. The expression of LC3 and beclin-1 (autophagy markers) is much higher in the LDLR KO and LDLR/LOX-1 DKO mice than in their counterpart WT and LOX-1 KO mice. Staining for mtDNA damage, TLR9 and inflammatory cell accumulation (CD45 and CD68) show similar pattern. The pictures are representative of multiple sections of aortas from different groups of mice (at least 5 in each group). Bar graphs represent data in mean \pm SD based on $3-5$ experiments. * $\mathrm{P}<0.05$ : LOX-1 KO vs. WT; ${ }^{\S} \mathrm{P}<0.05$ : Double KO vs. LDLR KO. Scale bars: $80 \mu \mathrm{m}$.

3-methyladenine enhanced TLR9 expression and treatment with rapamycin inhibited TLR9 expression. DNase II has been suggested to protect the heart from inflammation by digesting mtDNA and thereby block or attenuate the inflammatory response $\mathrm{e}^{6,7}$. In keeping with this postulate, inhibition of DNase expression by DNase II siRNA aggravated autophagy (enhanced expression of LC3 II and beclin-1) and TLR9 expression. These data were confirmed by immunocytochemistry and Western blotting. It has been suggested that mtDNA released from the dying cells into the extracellular space induces a TLR9-dependent inflammatory response in some cells of the immune system 6 . Oka et al. found that mtDNA that escapes degradation can trigger a similar response in the cell where the initial reaction started ${ }^{7}$. From the aforementioned observations, it can be concluded that LOX-1 mediated mtDNA damage increases its leakage into the cells, and induces autophagy. As leaked mtDNA exceeds the ability of autophagy to degrade, expression of TLR9 increases.

As reported previously ${ }^{3}$, VLDL- and LDL-cholesterol levels in plasma were much higher in the LDLR KO and LDLR/LOX-1 DKO mice than in the plasma of their counterpart WT and LOX-1 $\mathrm{KO}$ mice. Of interest, there was no difference in the cholesterol levels in LDLR KO and LDLR/LOX-1 DKO mice. However, extent of atherosclerosis (areas of sudanophilia) was much less in the LDLR/ LOX-1 DKO than in LDLR KO mice ${ }^{3}$. Presumably, either the oxLDL generation was much less in the LDLR/LOX-1 DKO than in LDLR KO mice or LOX-1 deletion resulted in reduced uptake of oxLDL resulting in attenuation of extent of sudanphilic areas. Previous studies from our group suggested a decrease in oxidant stress in the DKO mice as compared with LDLR KO mice.

We now extended the study by examining multiple sections of aorta from different groups of mice, and observed evidence for significant autophagy (expression of LC3 and beclin-1) and inflammation (expression of TLR9, CD45 and CD68) in the LDLR KO mice and these phenomena were attenuated by LOX-1 deletion. In keeping with the data from in vitro studies in HUVECs, mtDNA damage (and leakage) was extensive in LDLR KO mice and it was reduced by LOX-1 abrogation.

In conclusion, the present study confirmed our hypothesis that during oxidant stress damaged mtDNA leaks into cytoplasm, and mtDNA that escapes autophagy can trigger a TLR9-related inflammatory response. This phenomenon seems to occur in a LOX-1 dependent fashion, and may well be a basis of inflammation in atherosclerosis (Fig. 5). 


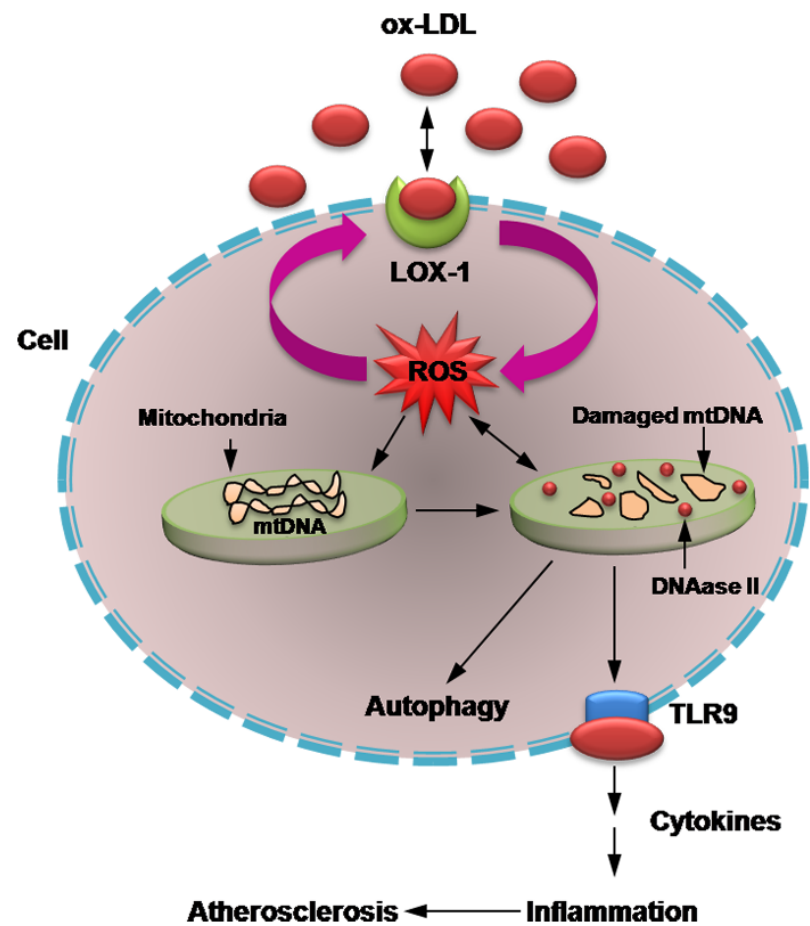

Figure $5 \mid$ Possible link between LOX-1-mediated mtDNA damage and autophagy. mtDNA that escapes autophagy induces a potent inflammatory response in atherosclerosis.

\section{Methods}

Cell culture. Primary human umbilical vein endothelial cells (HUVECs) were obtained from ATCC (Manassas, VA), and maintained in vascular cell basal medium. The cells were incubated at $37^{\circ} \mathrm{C}$ in a humidified atmosphere with $5 \% \mathrm{CO}_{2}$.

High TBAR ox-LDL ( 90 nmoles MDA/mg Protein) was purchased from Biomedical Technologies Inc., Stoughton, MA. Anti-human LOX-1 neutralizing antibody (LOX-1 Ab) JTX92 was a gift of Dr. T. Sawamura (Osaka, Japan). ROS inhibitor (Apocynin, $1 \mathrm{mmol} / \mathrm{l}$ ) was obtained from Sigma (St. Louis, MO). To examine the receptor specificity of ox-LDL action, HUVECs were pretreated with human LOX-1 blocking antibody $(10 \mu \mathrm{g} / \mathrm{ml})$ and then exposed to ox-LDL.

For mtDNA detection, HUVECs or aortic sections were incubated in PicoGreen (Molecular Probes, Carlsbad, CA) for $1 \mathrm{~h}$. Then fluorescent microscopy and flow cytometry were used to assess mtDNA expression.

Western blot. Details of Western blotting have been published recently ${ }^{4}$. The antibodies used were anti-rabbit LOX-1 (Abcam, CA), LC3 (Cell Signaling, Boston, MA), beclin-1 (Novus Biologicals, Littleton, CO), TLR9, CD45 and CD68 (last 3 from Santa-Cruz Biotechnology, Inc., CA).

Measurement of intracellular reactive oxygen species. Intracellular ROS was measured with the use of the fluorescent signal dihydroethidium (DHE), a cellpermeable indicator for ROS generation. Essentially, cells were cultured in 6-well plate and incubated with $10 \mu \mathrm{mol} / \mathrm{L}$ DHE in PBS for $30 \mathrm{~min}$. The ROS-mediated fluorescence was measured by flow cytometry (Becton Dickinson, Franklin Lakes, NJ), and the results were analyzed with the software WinMDI29, as described ${ }^{9}$.

DNase II siRNA transfection. In order to knock down DNase II, cells were transfected with small interfering RNA (siRNA) duplexes using siRNA transfection reagent (Santa Cruz, CA) as per manufacturer supplied protocols. Parallel aliquot of cells was transfected with sequence scrambled siRNA (ssc) duplexes as control.

Immunohistochemical analysis. HUVECs and aortic sections were stained with different antibodies using Mouse/Rabbit Specific HRP/DAB detection IHC kit (Abcam, San Francisco, CA) as per the manufacturer's instructions. Image J. v 1.46 (NIH, MD) was used to quantify immunohistochemical staining according to the manufacturer's instructions.

Animal protocol. The generation of LOX-1 KO and LOX-1/LDLR double KO mice on C57BL/6 background (also referred to as wild-type mice) has been described previously ${ }^{3}$. All animals were housed in the breeding colony at University of Arkansas for Medical Sciences, Little Rock, AR, USA. All male animals were given highcholesterol diet ( $4 \%$ cholesterol $/ 10 \%$ cocoa butter) for 18 weeks from the age of 6 weeks. All experimental procedures were performed in accordance with protocols approved by the Institutional Animal Care and Usage Committee, and conform to the
Guidelines for the Care and Use of Laboratory Animals published by the US National Institutes of Health.

Autophagy detection in HUVECs and rabbit thoracic aortas. Autophagy was detected by Western blot, fluorescent microscopy, immunohistochemistry (Premo $^{\mathrm{TM}}$ Autophagy Sensors (L3B-GFP) (Invitrogen, Grand Island, NY), and flow cytometry9.

Statistical analysis. Data from at least three independent experiments were used for statistical analysis. Results are shown as mean \pm SD. Multiple means were compared using a one-way analysis of variance (ANOVA). Paired Student's t-test was used to assess significant differences. $P$ value $<0.05$ was considered significant.

1. Sawamura, T. et al. An endothelial receptor for oxidized low-density lipoprotein. Nature 386, 73-77 (1997).

2. Chen, J. W. et al. Role of caspases in ox-LDL-induced apoptotic cascade in human coronary artery endothelial cells. Circ. Res. 94, 370-376 (2004).

3. Mehta, J. L. et al. Deletion of LOX-1 reduces atherogenesis in LDLR knockout mice fed high cholesterol diet. Circ. Res. 100, 1634-1642 (2007).

4. Li, D. \& Mehta, J. L. Upregulation of endothelial receptor for oxidized LDL (LOX-1) by oxidized LDL and implications in apoptosis of human coronary artery endothelial cells: evidence from use of antisense LOX-1 mRNA and chemical inhibitors. Arterioscler Thromb Vasc Biol 20, 1116-1122 (2000).

5. Madamanchi, N. R. \& Runge, M. S. Mitochondrial dysfunction in atherosclerosis. Circ Res 100, 460-473 (2007).

6. Konstantinidis, K. \& Kitsis, R. N. Cardiovascular biology: Escaped DNA inflames the heart. Nature 485, 179-180 (2012).

7. Oka, T. et al. Mitochondrial DNA that escapes from autophagy causes inflammation and heart failure. Nature 485, 251-255 (2012).

8. Okabe, Y., Okabe, Y., Kawane, K., Akira, S., Taniguchi, T. \& Nagata, S. Toll-like receptor-independent gene induction program activated by mammalian DNA escaped from apoptotic DNA degradation. J Exp Med 202, 1333-1339 (2005).

9. Ding, Z. et al. Degradation of heparan sulfate proteoglycans enhances oxidized-LDL-mediated autophagy and apoptosis in human endothelial cells. Biochem Biophys Res Commun 426, 106-11 (2012).

10. Levine, B., Mizushima, N. \& Virgin, H. W. Autophagy in immunity and inflammation. Nature 469, 323-335 (2011).

11. Evans, C. J. \& Aguilera, R. J. DNase II: genes, enzymes and function. Gene 322, 1-15 (2003).

12. Li, D. \& Mehta, J. L. Oxidized LDL, a critical factor in atherogenesis. Cardiovasc Res 68, 353-354 (2005).

13. Garber, K. Autophagy. Explaining exercise. Science 335, 281 (2012).

14. Collins, L. V., Hajizadeh, S., Holme, E., Jonsson, I. M. \& Tarkowski, A. Endogenously oxidized mitochondrial DNA induces in vivo and in vitro inflammatory responses. J Leukoc Biol 75, 995-1000 (2004).

15. Reis e Sousa, C. Immunology. Eating in to avoid infection. Science 315, 1376-1377 (2007).

16. Mizushima, N., Levine, B., Cuervo, A. M. \& Klionsky, D. J. Autophagy fights disease through cellular self-digestion. Nature 451, 1069-1075 (2008).

17. Nakahira, K. et al. Autophagy proteins regulate innate immune responses by inhibiting the release of mitochondrial DNA mediated by the NALP3 inflammasome. Nat Immunol 12, 222-230 (2011).

18. Krieg, A. M. CpG motifs: the active ingredient in bacterial extracts? Nat Med $\mathbf{9}$, 831-835 (2003).

19. Medzhitov, R. CpG DNA: security code for host defense. Nat Immunol 2, 15-16 (2001).

20. Ashley, N., Harris, D. \& Poulton, J. Detection of mitochondrial DNA depletion in living human cells using PicoGreen staining. Exp Cell Res 303, 432-446 (2005).

21. Saffran, H. A., Pare, J. M., Corcoran, J. A., Weller, S. K. \& Smiley, J. R. Herpes simplex virus eliminates host mitochondrial DNA. EMBO Rep 8, 188-193 (2007).

\section{Acknowledgements}

This study was supported in part by funds from the Department of Veterans Affairs.

\section{Author contributions:}

Z.D. and S.L. developed the concept of this study and performed the experiments; X.W. and M.K. performed immunohistochemical analyses; Y.D. helped with autophagy experiments; J.L.M. designed the study, analyzed the data and wrote the manuscript. All authors read and approved the final manuscript.

\section{Additional information}

Competing financial interests: The authors declare no competing financial interests. License: This work is licensed under a Creative Commons Attribution-NonCommercial-NoDerivs 3.0 Unported License. To view a copy of this license, visit http://creativecommons.org/licenses/by-nc-nd/3.0/

How to cite this article: Ding, Z. et al. Oxidant stress in mitochondrial DNA damage, autophagy and inflammation in atherosclerosis. Sci. Rep. 3, 1077; DOI:10.1038/srep01077 (2013). 